\title{
Overview of the Alliance for Cellular Signaling
}

\author{
Participating investigators and scientists of the Alliance for Cellular Signaling
}

${ }^{*}$ A full list of AfCS participating investigators and scientists appears at the end of this paper

The Alliance for Cellular Signaling is a large-scale collaboration designed to answer global questions about signalling networks. Pathways will be studied intensively in two cells - B lymphocytes (the cells of the immune system) and cardiac myocytes - to facilitate quantitative modelling. One goal is to catalyse complementary research in individual laboratories; to facilitate this, all alliance data are freely available for use by the entire research community.

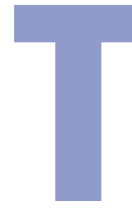

he availability of complete genomic sequences and the emerging technologies of proteomics inspire confidence that complex biological phenomena and systems can be understood completely. Such optimism is strengthened by rapidly expanding capabilities to undertake global analyses of biological systems, including the capacity to manipulate gene content and expression in mammalian cells and organisms, to detect protein-protein interactions, and to quantify the activities of macromolecules in vivo. Such understanding of complete systems implies (and demands) the capacity to predict quantitatively the altered behaviour of these systems that results from their genetic or environmental (including pharmacological) perturbation. We can foresee, rather than simply imagine, the construction of a virtual cell, and this accomplishment will eventually have an enormous impact on biomedical and pharmaceutical science.

We have launched a major new research initiative called the Alliance for Cellular Signaling (AfCS; www.signaling-gateway.org): a multidisciplinary, multi-institutional consortium to study cellular signalling systems. The overall goal of the AfCS is to understand as completely as possible the relationships between sets of inputs and outputs that vary both temporally and spatially. The same goal, stated from a slightly different perspective, is to understand fully how cells interpret signals in a context-dependent manner. How does a cell respond appropriately to one voice when it must listen simultaneously to many, and how does it alter this response in the context of other concurrent or recent signalling events? Answers to these questions will require identification of all the molecules that comprise the various signalling systems, assessment of time-dependent information flow through the systems in both normal and pathological states, and reduction of the mass of detailed data into a set of interacting models that describe cellular signalling.

The organization of the alliance was catalysed by an announcement by the National Institute of General Medical Sciences (NIGMS) of plans to fund large-scale collaborative projects to "enable the solution of major problems in biomedical research and to facilitate the next evolutionary stage of integrative biomedical science." NIGMS calls these 'glue' grants. The strategy of the AfCS includes a ten-year research plan with an annual budget of about US\$10,000,000. We have obtained the necessary funding from NIGMS, the National Institute of Allergy and Infectious Diseases, the National Cancer Institute, a consortium of pharmaceutical companies (Eli Lilly, Aventis, Johnson and Johnson, Novartis and (briefly) Chiron) and private donors (The Merck Genome Research Institute, the Agouron Institute and an anonymous philanthropist).

We plan to use these funds to answer overarching questions that cannot be approached by individual laboratories (Table 1). We are keenly aware of the fact that by acting alone, the alliance can acquire only a small fraction of the necessary information. A critical part of our strategy is thus to catalyse complementary research throughout the signalling community. We provide leads for pursuit by individual laboratories by disseminating all of our data publicly and promptly via the Internet. Information is released as soon as it has been verified, and neither AfCS investigators nor our sponsors have access to data before it is available to the public. We do experiments, but, importantly, we are ourselves an experiment in how collaborative science might be accomplished.

\section{Organization of the AfCS}

The so-called participating investigators of the AfCS comprise a group of around 50 scientists at about 20 different institutions, predominantly in the United States, with a few representatives from Canada and the United Kingdom. Participation is enhanced by communication and data sharing by videoconference using Internet2. (Internet2 is a university-led consortium whose goal is to create a leading-edge network capability for the national research community.)

The participating investigators are members of various committees or are directors of AfCS laboratories (Fig. 1; and see list of investigators at the end of this paper). A critical interaction is that between the laboratory directors and two 'system committees', representing the B lymphocyte and the cardiac myocyte (the two cells of primary interest to the AfCS). The system committees are charged with experimental planning and data interpretation, while the laboratory directors design and implement the experimental plan.

There is complete separation between the AfCS laboratories and the individual laboratories of the participating investigators. Participating investigators do not receive funding for their own laboratories, with the exception of modest funds that are provided for a few so-called 'bridging projects' (aimed at technology development). The eight AfCS laboratories are staffed by dedicated $\mathrm{PhD}$ scientists and technicians. The relatively small number of AfCS laboratories in which work is done minimizes experimental sources of variance as data are collected systematically.

Membership in the AfCS constitutes another level of participation in the venture. The role of the more than 1,500 members is described below.

\section{Selecting the cells to be studied}

We are committed to the tenet that much data must be gathered under standardized conditions on a small number of systems to permit the desired depth of quantitative understanding of signalling system behaviour. We have limited ourselves to two cell types - the B lymphocyte and the cardiac myocyte. The two cell types were not easy choices, indeed both pose enormous challenges, and agreement was reached only after a great deal of discussion. The primary criteria for choice of cells were that they should be mammalian with a sequenced genome, a criterion that drove us to the mouse; they should be nonmalignant and euploid, which led us to select cells that were as normal as possible and whose signalling systems had not been co-opted by malignant transformations; and they should be of requisite mass and homogeneity, offer 


\begin{tabular}{|c|c|c|}
\hline Question & Experiment & New technologies required \\
\hline $\begin{array}{l}\text { 1. How complex is signal processing in cells? Considering the set of ligands for } \\
\text { cell-surface receptors as a potential combinatorial code of inputs, how much of this input } \\
\text { complexity can a cell uniquely decode at its outputs? In other words, how big is the } \\
\text { AfCS problem, and can we experimentally bound the complexity of cellular signalling? }\end{array}$ & $\begin{array}{l}\text { Systematic single- and double-ligand } \\
\text { screens to classify output responses } \\
\text { and determine degree of functional } \\
\text { crosstalk between transduction pathways. }\end{array}$ & $\begin{array}{l}\text { Analytic methods to classify and } \\
\text { compare multi-dimensional data for } \\
\text { different ligand combinations. }\end{array}$ \\
\hline $\begin{array}{l}\text { 2. What is the structure of the whole signalling network? Is the connectivity } \\
\text { sparse or dense? }\end{array}$ & $\begin{array}{l}\text { Wholesale mapping of physiologically } \\
\text { relevant protein-protein and small- } \\
\text { molecule-protein interactions. }\end{array}$ & $\begin{array}{l}\text { High-throughput assays for inter- } \\
\text { molecular interaction in vivo, especially } \\
\text { in response to ligand stimulation. }\end{array}$ \\
\hline $\begin{array}{l}\text { 3. How much does network topology constrain signal processing capability? } \\
\text { How much of function is specified by the nature of connections rather than by the } \\
\text { specific biochemical constants of individual activities? Can a functional module be } \\
\text { defined and recognized simply by network topology? }\end{array}$ & $\begin{array}{l}\text { Perturbation methods (gain-of-function } \\
\text { and loss-of-function) coupled with high- } \\
\text { throughput functional assays to test the } \\
\text { role of potential network modules. }\end{array}$ & $\begin{array}{l}\text { Methods for reliably perturbing the } \\
\text { concentration (or specific activity) of } \\
\text { signalling proteins in vivo, both singly } \\
\text { and in combinations. }\end{array}$ \\
\hline $\begin{array}{l}\text { 4. Can functional modules be abstracted mathematically? Can we make physical } \\
\text { models of the network modules that explain and predict the input-output relations of } \\
\text { signalling subsystems? Can we identify the basic computations being done by these } \\
\text { modules? What is the 'device physics' of biological signalling components? }\end{array}$ & $\begin{array}{l}\text { Mathematical modelling of network } \\
\text { modules coupled with experimental } \\
\text { testing. }\end{array}$ & $\begin{array}{l}\text { New mathematical methods to model } \\
\text { the behaviour of signalling systems. } \\
\text { Approaches to improve the interface } \\
\text { between theorists and experimentalists. }\end{array}$ \\
\hline $\begin{array}{l}\text { 5. What are the dynamics of the signalling network? Can we visualize how } \\
\text { perturbations to the signalling network (such as ligand application) propagate through } \\
\text { the network as changes in functional activities? This represents the flow and } \\
\text { transformation of information during signalling events. }\end{array}$ & $\begin{array}{l}\text { Follow the kinetics of signalling through } \\
\text { a set of nodes in the signalling network } \\
\text { that comprise a representative sampling } \\
\text { of the sites of information processing. }\end{array}$ & $\begin{array}{l}\text { Methods for monitoring protein activities } \\
\text { with high temporal and spatial resolution } \\
\text { in cells. }\end{array}$ \\
\hline $\begin{array}{l}\text { 6. Why is the network the way it is? What general thermodynamic principle operates } \\
\text { on evolved systems to make the observed solutions to information processing the } \\
\text { chosen ones? What is being optimized so that the signalling network looks and } \\
\text { behaves the way it does? }\end{array}$ & Unknown for now. & Unknown for now. \\
\hline
\end{tabular}

the potential to manipulate gene expression, and possess interesting biological properties regulated by interacting signalling systems.

The significant advantages and the disadvantages of these cells are described in the accompanying articles (pages 708-710 and 712-714). There is no single cell type that satisfies all of our desiresit is difficult to find all wanted attributes and bring all necessary technologies to a single system - and it remains to be seen if we have struck a set of manageable compromises.

\section{Experimental strategy}

Our goal is to understand the cellular signalling networks that convert a set of time-varying inputs into physiologically relevant outputs. This requires identification and localization of all the relevant molecules, definition of the physical interactions between these molecules, and quantitative assessment of the physiological significance of these interactions as information flows through the system. Furthermore, it is critical that this flow of information be evaluated under conditions where interactions between combinations of inputs can be assessed, as this is the only way to probe the level of complexity of the system. These conceptual requirements dictate three broad and temporally ordered (but overlapping) experimental approaches, outlined in Table 2.

\section{The parts list}

What signalling proteins are expressed in the cells of interest, where are they located within these cells, and do they move within these cells in response to the flow of regulatory information? To answer these questions we must conduct broad screens to detect interactions between proteins and to identify players that do not yet appear on the AfCS protein list. We are conducting yeast two-hybrid screens using AfCS proteins as bait in collaboration with Myriad Genetics. Results are posted on the alliance website as they are received and should represent a valuable source of leads for pursuit by individuals in the signalling research community. We hope to launch other proteinand DNA-based screens for relevant interactions in the near future.

\section{Ligand screens}

We need to know what ligands will activate signalling pathways in the chosen cells, what combinations of ligands will reveal interactions between signalling pathways, and what downstream responses are reliable indicators of signalling activity. The single and multiple ligand screens are designed to provide this information. B cells and cardiac myocytes are being exposed to a large number of ligands chosen according to information from the literature. We are sampling cellular responses to each ligand with a collection of assays that should reveal the nature and breadth of the response, including second messenger concentrations, alterations in protein phosphorylation, patterns of gene expression and, in time, concentrations of many cellular lipids. We anticipate compilation of a list of 20 or more active ligands for each cell type. The single ligand screen will tell us if any one ligand is truly the same as another (for example, how much do the agonists that work on the distinct $\mathrm{G}_{\mathrm{i}}$-coupled chemokine receptors of $\mathrm{B}$ cells differ from each other) and will reveal responses to be targeted for careful quantification when we return to measure information flow through the entire system.

Measurements of responses to combinations of ligands will reveal interactions between pathways. Interaction is revealed by energetic (logarithmic) non-additivity. We will thus evaluate the additivity of the log fraction (or multiple) of the output. The combinations of inputs that yield the most extensive deviations from energetic additivity will be carried forward for rigorous analysis. We cannot fully sample the multidimensional space of two-way ligand combinations (various concentrations, time points, order of additions, and so on), to say nothing of higher-order combinations. Additionally, we have no idea of the density of interactions that we might uncover, but some estimate of this parameter will provide the first at least semi-systematic estimate of the level of complexity of cellular signalling systems.

\section{Perturbational analysis}

With parts lists and ligands in hand, we will undertake a perturbational strategy to construct an epistasis map. Such approaches are as old as pharmacology and genetics, where they were born. Most investigators would likely agree that pharmacological manipulation of a system (be it called chemical genetics or not) has more to offer (in terms of speed and reversibility) when cell-permeant chemicals of high specificity are available. But the number of adequate chemical perturbants is unfortunately small, and we must rely heavily on nucleic acid-based techniques to manipulate gene expression to achieve either loss or gain of function. RNA interference is particularly promising, and successful application of this technology would greatly improve the quality of our effort. Antisense approaches are also being pursued. Just as we will apply combinations of ligands to our experimental cells, it will become equally important to use combinations of perturbants.

\section{Quantitative determination of information flow}

The final experimental phase of the analysis will be the quantitative determination of functional interactions among the signalling molecules. This ambitious undertaking will start when the system committees decide that the identification of new signalling 


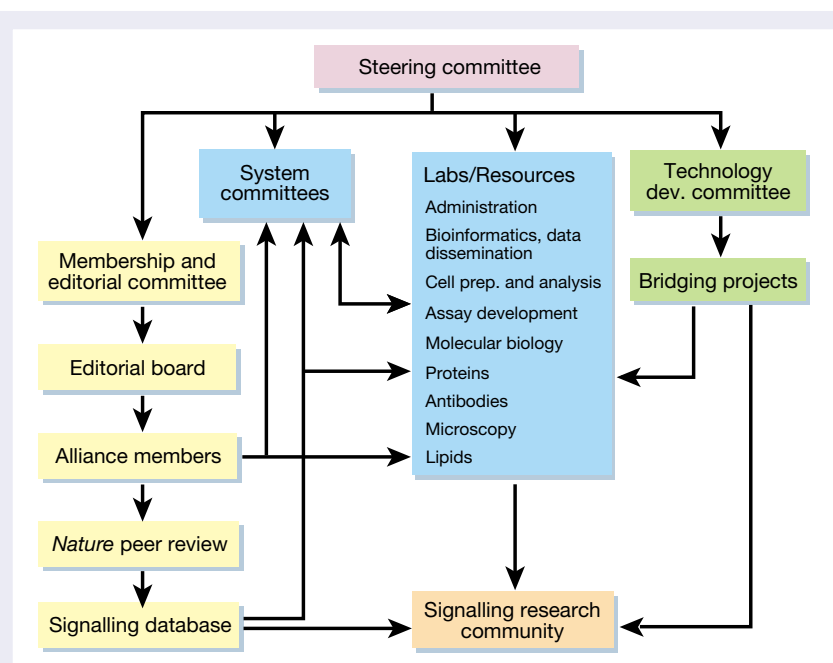

Figure 1 AfCS organizational chart.

molecules and their interactions is approaching saturation. The data from this effort will allow us to attain our goal of performing the first molecular analysis of how a signalling network actually works.

For this goal, it will almost certainly be inappropriate to adopt the traditional reductionist approach of simply measuring in vitro all the $K_{\mathrm{d}}, K_{\mathrm{m}}$ and $V_{\max }$ values, cooperativity constants, rate constants and molar concentrations for all molecules involved. One reason is that it is unlikely that any artificial solution (medium) will allow approximation of the physiological magnitude of these values. Multiple specific interactions of a single molecule in the cell will also be impossible to duplicate in vitro. And determination of the vast array of values that the strict reductionist approach demands will probably be technologically impossible in the foreseeable future, and their manipulation to yield a model of cell function would be daunting.

An alternative approach to quantifying the matrix of regulatory interactions in the cell is to measure functional interactions on essentially arbitrary scales for each molecule that we test, and then to link fractional output (fractional activation, activity and binding) of one molecule with its fractional effect on those with which it interacts. In general, we will stimulate and/or inhibit the activity of each molecule $p$ over a defined range and measure consequent changes in the activities (or other parameters) of all molecules $q_{n}$ with which $p$ interacts directly in a functionally important way. By asking how much of the maximal activity of $p$ does it take to produce fractional activation of $q_{1}, q_{2}, \ldots, q_{n}$, we hope to arrive at a set of linkage functions that should in principle be determined by fractional activation of receptors by ligands and be interpretable as fractional physiological outputs.

What are the experimental parameters that will give us these fractional activities? Each will depend on the type of molecule measured. For $\mathrm{Ca}^{2+}$, concentration is adequate, and appropriate assays are already available. For a protein phosphatase, activity will vary with substrates, and fractional activation towards one substrate will not necessarily equal that towards another, as modulation can change specificity. Conceptually, any 'reporter' will do, but reporters must be closely linked to the molecules whose activities they describe or their information will be degraded (filtered) by other interactions in the signalling network. Development of appropriate in situ signalling assays is one of the more rapidly evolving technologies, and the alliance will draw on the leaders in this field. Spectroscopic outputs are clearly preferable, and product traps for enzymes that synthesize stable, low-molecular-weight molecules are also desirable.

\section{Data modelling and network analysis}

The alliance is built on two independent developments that have become prominent during the past decade: the success of molecular biology in developing tools to identify the component parts of biological systems and their interactions, and the development of mathematical and computational tools for the analysis and design of complex engineering systems.

The first of these advances provides measurement technology to yield quantitative assessments of the state of components of biological networks (for example, transcripts, small ions, labelled proteins and cell shapes) that begin to equal the tools used for the analysis of other engineering networks. In fact, the early stages of the alliance's efforts are devoted to the further identification of the components of cellular signalling networks and the quantitative measurement of their input-output behaviours and regulation. But appreciation of the biological implications of these measurements will be largely impenetrable without data analysis and modelling tools designed specifically for biological network systems. Thus the final stages of the alliance's activity must address the 'complex systems' aspects of biology, building on methods from systems engineering, computer science, control theory, circuit design and dynamical systems.

The current charge of the alliance's network analysis team is to produce the necessary theoretical and computational framework to achieve these final goals. This requires close interactions with the database and informatics teams to create data structures and tables appropriate for network analysis, and with the system committees to assure that the experimental procedures yield data that are appropriate for network prediction and analysis. We must create tools for organization and analysis of these data and then build static and dynamic models of system function that can be validated experimentally. These models can then be used to design practical biomedical strategies for control of these pathways and their associated disease states. Thus, this team must forge the necessary tight cycle of experiment, theory and computation that is the hallmark of the analysis and engineering of networks in other fields of inquiry, and the alliance has been structured to permit this type of interaction.

\section{Establishment of a signalling database}

The AfCS will sponsor and coordinate the establishment of a signalling database that will be an invaluable resource for the entire signalling

\section{Table 2 An overview of the experimental strategy of the AfCS}

Identify and localize the relevant molecules

1. Define basic scope, including pathways of interest and an initial list of molecules. (I) ${ }^{\star}$

2. What molecules are present? Large-scale transcriptional profiling. (I)

3. Quantify proteins of interest (using immunoblotting, ligand binding). (I)

4. Localize proteins of interest (by microscopy, subcellular fractionation). (l)

5. Monitor movements of proteins, preferably quantitatively and in real time (for example, using GFP fusions); also a quantitative intermediate endpoint. (I)
Define and expand the molecule list

1. Broad screens to detect interactions between proteins The goal is to define and expand the list of molecular players, and to identify 'all' of the interactions among the components. The research community at large will define the physical and physiological validity of these interactions. The alliance will define the further physiological validity and the quantitative physiological significance of these interactions in its two cellular systems. (I)
Define the flow of information

1. Choose the inputs using broad assays to eliminate redundancy and detect interactions. (I)

2. Define intermediate endpoints. $(I, L)$

3. Choose the outputs. $(I, L)$

4. Measure intermediate endpoints and outputs as a function of inputs (for example, time, concentration, combinations) while altering functional concentrations of components (the molecule list, singly and in combinations) pharmacologically and 'genetically'. (L)

5. Define quantitative relationships between activity states of interacting components; assays in real time in vivo and snap shots of intracellular activity. (L)

6. Develop and test models. (L) 
research community. The central element of this database will be the 'Molecule Pages', which will aim to represent every protein on the AfCS list (see article on pages 716-717). These will be standardized documents that each contain a wealth of literature-derived information about a given molecule; additional information from the alliance laboratories will be incorporated in time. Linkages will be provided between Molecule Pages to maps of signalling pathways as they are developed and refined, to the original literature, and to other databases.

Molecule Pages will be prepared (and updated) by AfCS members and will be peer reviewed, the entire process being supervised by an editorial board and Nature. We believe strongly that the collection and interpretation of the considerable amount of information about a given protein and its entry into a complex object-relational database is a substantial scholarly effort - at least as important as a conventional written review because of the powerful nature of the database itself. Given peer review of these Molecule Pages and the important collaboration between the AfCS and Nature Publishing Group, we believe that AfCS Molecule Pages should and will have the cachet of a major publication in Nature or a similar high-profile journal. Members will be chosen particularly for their expertise about specific molecules and pathways, and applications for membership have been and will continue to be encouraged publicly via our website.

\section{Socialistic aspects of the AfCS concept}

The alliance is itself an experiment in collaborative science. There have been relatively few large-scale collaborations in basic biology. It is a young science that has flourished to date in the hands of individual investigators, directing relatively small laboratories. But the scientific questions posed by the AfCS are not suited to the traditional distributed approach to experimental biology.

Are there impediments to large-scale collaboration? There are significant nuisances, but they pale by comparison with the potential gain. Obvious difficulties imposed by distance can be largely circumvented with modern communication systems; the AfCS does not function without the Internet. Human and career issues are significant -scientists are no less egocentric or competitive than others. We may be endowed with exuberant senses of curiosity, but we can lay no collective claim to sainthood. In addition, the academic reward system compounds the problem, and the AfCS does not provide a wealth of conventional academic rewards.

It is essential that we distribute data publicly and promptly via the Internet; publications of experimental observations (but not analyses) are thus largely superfluous. The vast majority of AfCS funding is spent in the AfCS laboratories to optimize precision and standardization of data collection. The classical promotion and tenure system needs adjusting to recognize an individual's contributions to group efforts if large-scale biology is to come of age. Physicists have learned the art of large-scale collaboration because of collective needs for extremely expensive facilities. Biologists will learn the same art because of the overwhelming complexity of the problems to be solved.

Our challenge is substantial, and it can be met only by the coordinated and cooperative efforts of many, in roles ranging from preparation of Molecule Pages by our members, to donation of significant amounts of time and energy by our participating investigators, to full-time employment by AfCS laboratory scientists and technicians. These contributions will benefit the entire field enormously and expand the opportunities for everyone. Those involved with this project see the need clearly and bring their own motivation to the project. We are excited by the idea that key questions about cellular information processing can be answered by a collective approach and that such understanding will produce new paradigms to guide further studies in individual laboratories. The alliance offers this opportunity in a unique way, but only to those who are willing to donate a part of themselves to the group. We need socialistic science practised by strong individualists. Practical? We believe it is.

doi:10.1038/nature01304

\section{Acknowledgements}

This article was written by R. Taussig, R. Ranganathan, E. M. Ross and A. G. Gilman (University of Texas Southwestern Medical Center) on behalf of the participating investigators and scientists of the AfCS. R.R. is an Associate Investigator of the Howard Hughes Medical Institute.

\section{Participating investigators and scientists of the AfCS}

Steering Committee: Alfred G. Gilman ${ }^{1}$ (Chair), Melvin I. Simon ${ }^{6}$ (Vice Chair), Henry R. Bourne ${ }^{9}$, Bruce A. Harris ${ }^{13}$, Rochelle Long ${ }^{14}$, Elliott M. Ross ${ }^{1}$, James T. Stull' ${ }^{2}$, Ronald Taussig ${ }^{1}$

B Lymphocyte System Committee: Henry R. Bourne ${ }^{9}$ (Chair), Adam P. Arkin ${ }^{15}$, Melanie H. Cobb ${ }^{1}$ Jason G. Cyster ${ }^{10}$, Peter N. Devreotes ${ }^{16}$, James E. Ferrell ${ }^{17}$, David Fruman ${ }^{20}$, Michael Gold ${ }^{21}$, Arthur Weiss ${ }^{11}$

Cardiac Myocyte System Committee: James T. Stull ${ }^{2}$ (Chair), Michael J. Berridge ${ }^{22}$, Lewis C. Cantley ${ }^{23}$ William A. Catterall ${ }^{24}$, Shaun R. Coughlin ${ }^{12}$, Eric N. Olson ${ }^{5}$, Temple F. Smith ${ }^{25}$

External Advisory Committee: Joan S. Brugge ${ }^{26}$ (Chair), David Botstein ${ }^{18}$, Jack E. Dixon ${ }^{27}$, Tony Hunter $^{28}$, Robert J. Lefkowitz ${ }^{29}$, Anthony J. Pawson ${ }^{31}$, Paul W. Sternberg ${ }^{7}$, Harold Varmus ${ }^{32}$

Bioinformatics and Data Coordination Laboratory: Shankar Subramaniam ${ }^{33}$ (Director), Robert S. Sinkovits ${ }^{33}$, Joshua Li ${ }^{33}$, Dennis Mock $^{33}$, Yuhong Ning ${ }^{33}$, Brian Saunders ${ }^{33}$

Cell Preparation and Analysis Laboratory: Paul C. Sternweis ${ }^{1}$ (Director), Donald Hilgemann ${ }^{2}$ and Richard H. Scheuermann ${ }^{3}$ (Assoc. Directors), Dianne DeCamp ${ }^{1}$, Robert Hsueh ${ }^{1}$, Keng-Mean Lin ${ }^{1}$, Yan $\mathrm{Ni}^{1}$

Laboratory for Development of Signaling Assays: William E. Seaman ${ }^{10,11,34}$ (Director), Paul C. Simpson ${ }^{11,12,35}$ (Co-Director), Timothy D. O'Connell ${ }^{35}$, Tamara Roach ${ }^{34}$

Molecular Biology Laboratory: Melvin I. Simon ${ }^{6}$ (Director), Sangdun Choi ${ }^{6}$, Pamela Eversole-Cire, Iain Fraser ${ }^{6}$

Protein Chemistry Laboratory: Marc C. Mumby ${ }^{1}$ (Director), Yingming Zhao ${ }^{4}$ (Consultant), Deirdre Brekken ${ }^{1}$, Hongjun Shu ${ }^{1}$

Microscopy Laboratory: Tobias Meyer ${ }^{19}$ (Director), Grischa Chandy ${ }^{19}$, Won Do Heo ${ }^{19}$, Jen Liou ${ }^{19}$, Nancy O'Rourke $^{19}$, Mary Verghese ${ }^{19}$

Antibody Laboratory: Susanne M. Mumby ${ }^{1}$ (Director), Heping Han

Lipidomics Laboratory: H. Alex Brown ${ }^{36}$ (Director), Jeffrey S. Forrester ${ }^{36}$, Pavlina Ivanova ${ }^{37}$, Stephen B. Milne $^{38}$

Membership and Editorial Committee: Patrick J. Casey ${ }^{30}$ (Chair), T. Kendall Harden ${ }^{38}$

Bridging Project Investigators: Adam P. Arkin ${ }^{15}$, John Doyle ${ }^{8}$, Martha L. Gray ${ }^{39}$, Tobias Meyer ${ }^{19}$, Stephen Michnick ${ }^{41}$, Martin A. Schmidt ${ }^{40}$, Mehmet Toner $^{42}$, Roger Y. Tsien ${ }^{43}$

Data Analysis: Madhusudan Natarajan ${ }^{1}$, Rama Ranganathan ${ }^{1}$, Gilberto R. Sambrano ${ }^{12}$

Departments of ${ }^{1}$ Pharmacology, ${ }^{2}$ Physiology, ${ }^{3}$ Pathology, ${ }^{4}$ Biochemistry, and ${ }^{5}$ Molecular Biology and Oncology, University of Texas Southwestern Medical Center, Dallas, Texas 75390, USA $;{ }^{6}$ Biology Division, 147-75, ${ }^{7}$ Department of Biology, Howard Hughes Medical Institute, and ${ }^{8}$ Electrical Engineering, California Institute of Technology, Pasadena, California 91125, USA; Departments of ${ }^{9}$ Cellular \& Molecular Pharmacology, ${ }^{10}$ Microbiology and Immunology, ${ }^{11}$ Medicine, and ${ }^{12}$ Cardiovascular Research Institute, University of California at San Francisco, San Francisco, California 94143, USA; ${ }^{13}$ Aventis Pharmaceuticals, Bridgewater, New Jersey 08807, USA $;{ }^{14}$ Pharmacology, Physiology \& Biological Chemistry Division, NIGMS, NIH, Bethesda, Maryland 20892, USA; ${ }^{15}$ Departments of Bioengineering \& Chemistry, University of California, E.O. Lawrence Berkeley Natl. Laboratory, Berkeley, California 94720, USA; ${ }^{16}$ Department of Cell Biol. \& Anatomy, Johns Hopkins University, Baltimore, Maryland 21205, USA ${ }^{17}$ Center for Clinical Science Research, Departments of ${ }^{18}$ Genetics and ${ }^{19}$ Molecular Pharmacology, Stanford University School of Medicine, California 94305, USA $;{ }^{20}$ Department of Molecular Biology \& Biochemistry, University of California, Irvine, California 92697, USA; ${ }^{21}$ Deptartment of Microbiology and Immunology, University of British Columbia, Vancouver, British Columbia, V6T 1 Z3 Canada; ${ }^{22}$ The Babraham Institute, Cambridge CB2 4AT, UK; ${ }^{23}$ Departments of Cell Biology \& Medicine, Harvard Medical School, Beth Israel Deaconess Medical Center Boston, Massachusetts 02215, USA: ${ }^{24}$ Department of Pharmacology, University of Washington School of Medicine, Seattle, Washington 98195, USA ${ }^{25}$ Boston University, BMERC, Boston, Massachusetts 02215, USA; ${ }^{26}$ Department of Cell Biology, Harvard Medical School, Boston, Massachusetts 02115, USA; ${ }^{27}$ Department of Biol. Chem., University of Michigan Medical School, Ann Arbor, Michigan 48109, USA; ${ }^{28}$ The Salk Institute for Biological Studies, San Diego, California 92186, USA; ${ }^{29}$ Howard Hughes Medical Institute, and ${ }^{30}$ Department of Pharmacology \& Cancer Biology, Duke University Medical Center, Durham, North Carolina 27710, USA; ${ }^{31}$ Mount Sinai Hospital, Toronto, Ontario M5G IX5, Canada; ${ }^{32}$ Memorial Sloan Kettering Cancer Center, New York 10021, USA; ${ }^{33}$ San Diego Supercomputer Center, University California, San Diego, La Jolla, California 92037, USA; Department of ${ }^{34}$ Rheumatology and ${ }^{35}$ Cardiology, Veteran Affairs Medical Center, San Francisco, California 94121, USA; ${ }^{36}$ Department of Pharmacology, Vanderbilt University, Nashville, Tennessee 37232, USA $;^{37} 118$ Hatherley Road, Syracuse, New York 13224, USA ${ }^{38}$ Department of Pharmacology, School of Medicine, The University of North Carolina, Chapel Hill, North Carolina 27599, USA; ${ }^{39}$ Department of Electrical and Medical Engineering, Harvard-MIT Division of Health Sciences \& Technology, and ${ }^{40}$ Department of Electrical Engineering \& Computer Science, Massachusetts Institute of Technology, Cambridge, Massachusetts 02139, USA ${ }^{41}$ Department of Biochemistry, University of Montreal, Quebec, Canada H3C $3 J 7 ;{ }^{42}$ Shriners Burn Hospital, Boston, Massachusetts 02114, USA; ${ }^{43}$ Howard Hughes Medical Institute, University of California at San Diego, La Jolla, California 92093, USA 